Mathematical Modelling and Analysis

Volume 11 Number 1, 2006, pages 87-94

(c) 2006 Technika ISSN 1392-6292

\title{
GENERALIZED EULER-KNOPP METHOD AND CONVERGENCE ACCELERATION
}

\author{
O. MERONEN and I. TAMMERAID
}

Department of Mathematics, Tallinn University of Technology

Tallinn, 19086, Estonia

E-mail: olgam@tpu.ee; itammeraid@edu.ttu.ee

Received September 27, 2005; revised January 26, 2006

\begin{abstract}
New propositions on $\lambda$-boundedness for generalized Euler-Knopp method of summability $(\mathcal{E}, T)$, where $T$ is a linear bounded operator from Banach space $X$ into $X$, are proved. Using these results are verified a proposition on convergence acceleration by $(\mathcal{E}, T)$ and a Tauberian remainder theorem for $(\mathcal{E}, T)$.

Key words: convergence acceleration, summability methods, Tauberian remainder theorems
\end{abstract}

\section{Introduction and Lemmas}

Let $X, Y$ be Banach spaces and $\mathcal{L}(X, Y)$ be a space of all linear bounded operators from Banach space $X$ into $Y$. A sequence $x=\left(\xi_{k}\right)\left(\xi_{k} \in X\right)$ is called $\lambda$ - bounded if

$$
\exists \lim \xi_{k}=\xi \wedge \beta_{k}=\lambda_{k}\left(\xi_{k}-\xi\right) \wedge \beta_{k}=O(1),
$$

whereas $\lambda=\left(\lambda_{k}\right)$ with $0<\lambda_{k} \nearrow$.

Let $m_{X}^{\lambda}$ be the set of all $\lambda$-bounded sequences. A sequence $x=\left(\xi_{k}\right)$ is called summable (see [20] and [8]) by a generalized method $\mathcal{A}=\left(A_{n k}\right)$, $A_{n k} \in \mathcal{L}(X, Y)$ if $y=\left(\eta_{n}\right)$ with

$$
\eta_{n}=\sum_{k=0}^{\infty} A_{n k} \xi_{k}
$$

is convergent. Let $\mu=\left(\mu_{k}\right)$ with $0<\mu_{k} \nearrow$. The transformation $\mathcal{A}$ is called preserving $\lambda$-boundedness (see $[6]$ and also $[1,2,9,14]$ ) if

$$
\mathcal{A} m_{X}^{\lambda} \subset m_{Y}^{\lambda} \text {. }
$$

The transformation $\mathcal{A}$ is called accelerating $\lambda$-boundedness if 


$$
\mathcal{A} m_{X}^{\lambda} \subset m_{Y}^{\mu}
$$

with $\lim \mu_{k} / \lambda_{k}=\infty$. A method $\mathcal{A}=\left(A_{n k}\right)$ with $A_{n k} \in \mathcal{L}(X, X)$ is called regular if $\mathcal{A} c_{X} \subset c_{X}$ and

$$
\lim _{n} \eta_{n}=\lim _{k} \xi_{k}
$$

while $c_{X}$ is a set of convergent sequences with $\xi_{k} \in X$ and $\eta_{n}$ is defined by (1.1). We denote by $I$ and $\theta$ the identity and zero operator on any Banach space, respectively.

Kornfeld (see [10]) proved that any regular numerical method of summability can not universally accelerate the convergence. In [6] it is proved that any regular triangular generalized method $\mathcal{A}$ satisfying the condition

$$
\sum_{k=0}^{n} A_{n k}=I
$$

can not accelerate the convergence. Regardless of this fact in applied mathematics linear triangular methods are used to accelerate the convergence (see [16]). Such acceleration is possible in some subsets of $m_{X}^{\lambda}$. The present article is a sequel to the inquiries $[6,16,17,18,19]$. Main results of convergence acceleration using nonlinear methods are presented in [4].

Let us denote by $(\mathcal{E}, T)$ or shortly $\mathcal{E}$ the generalized Euler-Knopp method of summability defined (see $[3,12,18])$ by

$$
E_{n k}= \begin{cases}\left(\begin{array}{l}
n \\
k
\end{array}\right) T^{k} & (I-T)^{n-k}, \quad(k=0,1, \ldots, n), \\
\theta, & (k>n)\end{cases}
$$

where $T \in \mathcal{L}(X, X)$, while $T \neq \theta$ and $T^{0}=I$.

To prove our propositions we use the following Lemmas. It is easy to prove Lemma 1 and Corollaries 1 and 2 in the same way as the analogical assertions (see [2]) are proved in the case of number matrices.

Lemma 1. The product of generalized Euler-Knopp methods $(\mathcal{E}, U)$ and $(\mathcal{E}, V)$, where $U, V \in \mathcal{L}(X, X)$, is Euler-Knopp method $(\mathcal{E}, U V)$.

Corollary 1. If $T \in \mathcal{L}(X, X)$ and $m \in \mathbf{N}$, then $(\mathcal{E}, T)^{m}=\left(\mathcal{E}, T^{m}\right)$

Corollary 2. If $T \in \mathcal{L}(X, X)$ and $T^{-1} \in \mathcal{L}(X, X)$, then the $\left(\mathcal{E}, T^{-1}\right)$ is the inverse of the method $(\mathcal{E}, T)$.

Let $\xi_{n} \in X$ and $x^{(\nu)}=\left(\xi_{k}^{(\nu)}\right)$, while

$$
\xi_{n}^{(0)}=\xi_{n}, \quad \xi_{n}^{(\nu+1)}=\sum_{k=0}^{n} E_{n k} \xi_{k}^{(\nu)} \quad\left(\nu \in \mathbf{N}_{0}\right)
$$

$E_{n k}$ are determined by the use of (1.4) and $x^{(\nu+1)}=\mathcal{E} x^{(\nu)}$.

Analogically as in the case of number matrices (see [15]) it is possible to prove the next Lemma. 
Lemma 2. If $(\mathcal{E}, T)$ is a generalized Euler-Knopp method of summability defined by (1.4), and sequence $x^{(\nu)}=\left(\xi_{k}^{(\nu)}\right)$ is defined by (1.5), then

$$
\Delta \xi_{n}^{(\nu+1)}=\sum_{k=0}^{n} \frac{k}{n}\left(\begin{array}{l}
n \\
k
\end{array}\right) T^{k}(I-T)^{n-k} \Delta \xi_{k}^{(\nu)},
$$

while

$$
\Delta \xi_{n}^{(\nu)}= \begin{cases}\xi_{n}^{(\nu)}-\xi_{n-1}^{(\nu)} & (n \in \boldsymbol{N}), \\ \xi_{n}^{(\nu)} & (n=0) .\end{cases}
$$

Lemma 3. (see [12]). Method $(\mathcal{E}, T)$ is regular if and only if

$$
\|T\|+\|I-T\| \leq 1, \quad\|I-T\|<1 .
$$

Remark 1. As

$$
1=\|I\|=\|T+(I-T)\| \leq\|T\|+\|(I-T)\|,
$$

then the first inequality in (1.7) implies

$$
\|T\|+\|I-T\|=1 .
$$

Remark 2. If $T=c I$ with $0<c \leq 1$, then the method $(\mathcal{E}, T)$ is regular.

Lemma 4. (see [6]). Let us have $\mathcal{A}=\left(A_{n k}\right), A_{n k} \in \mathcal{L}(X, Y)$, and $e_{X}(\varsigma):=$ $(\varsigma, \varsigma, \varsigma, \ldots)$ with $\varsigma \in X$. If

$$
\exists \lim _{n} A_{n k}=A_{k} \quad\left(k \in \boldsymbol{N}_{0}\right)
$$

in norm, then the conditions

$$
\begin{aligned}
& \mathcal{A} e_{X}(\varsigma) \in m_{Y}^{\mu} \quad(\varsigma \in X), \quad \sum_{k} \lambda_{k}^{-1}\left\|A_{k}\right\|<\infty, \\
& \mu_{n} \sum_{k} \lambda_{k}^{-1}\left\|A_{n k}-A_{k}\right\|=O(1)
\end{aligned}
$$

are sufficient for the inclusion (1.2).

\section{Convergence Preservation and Convergence Acceleration}

Proposition 1. (see also [18]). If $X$ is a Banach space and $(\mathcal{E}, T)$ is determined by (1.4), then the conditions (1.7) and

$$
\mu_{n}\|I-T\|^{n} \sum_{k=0}^{n}\left(\begin{array}{l}
n \\
k
\end{array}\right) \frac{1}{\lambda_{k}}\left(\frac{\|T\|}{\|I-T\|}\right)^{k}=O(1)
$$

are sufficient for the inclusion

$$
(\mathcal{E}, T) m_{X}^{\lambda} \subset m_{X}^{\mu}
$$


Proof. Let us verify the conditions of the Lemma 4 by fixing $\mathcal{A}=\mathcal{E}$. By Lemma 4 the conditions (1.7) are sufficient for the regularity of the method $\mathcal{E}$. As $\mathcal{E}$ is regular, then (see [12]) $A_{k}=\theta\left(k \in \mathbf{N}_{0}\right)$. The second condition (1.9) follows from $A_{k}=\theta\left(k \in \mathbf{N}_{0}\right)$. Using (1.1) and (1.4) we get for $\varsigma \in X$ that

$$
\eta_{n}=\sum_{k=0}^{n}\left(\begin{array}{l}
n \\
k
\end{array}\right) T^{k}(I-T)^{n-k} \zeta=(T+(I-T))^{n} \varsigma=I \varsigma=\varsigma \quad\left(n \in \mathbf{N}_{0}\right) .
$$

So we have

$$
\begin{aligned}
& \eta=\lim _{n} \eta_{n}=\lim _{n} \varsigma=\varsigma, \\
& \mu_{n}\left(\eta_{n}-\eta\right)=\mu_{n}(\varsigma-\varsigma)=0, \quad \mathcal{E} e_{X}(\varsigma) \in m_{X}^{\mu} \quad(\varsigma \in X) .
\end{aligned}
$$

That means the first condition (1.9) is satisfied. As $A_{k}=\theta\left(k \in \mathbf{N}_{0}\right)$, by the second condition (1.7) we get

$$
\begin{aligned}
\left\|A_{n k}-A_{k}\right\| & =\left\|A_{n k}\right\|=\left\|\left(\begin{array}{l}
n \\
k
\end{array}\right) T^{k}(I-T)^{n-k}\right\| \\
& \leq\left(\begin{array}{l}
n \\
k
\end{array}\right)\|T\|^{k}\|(I-T)\|^{n-k} \stackrel{n \rightarrow \infty}{\rightarrow} 0 .
\end{aligned}
$$

So the condition (1.8) is satisfied. The condition (1.10) follows from the condition (2.1). The conditions of Lemma 4 are satisfied and from (1.2) we get (2.2). This completes the proof.

Corollary 3. The conditions (1.7) and

$$
\lambda_{n}\|I-T\|^{n} \sum_{k=0}^{n}\left(\begin{array}{l}
n \\
k
\end{array}\right) \frac{1}{\lambda_{k}}\left(\frac{\|T\|}{\|I-T\|}\right)^{k}=O(1)
$$

are sufficient for

$$
(\mathcal{E}, T) m_{X}^{\lambda} \subset m_{X}^{\lambda}
$$

Corollary 4. If

$$
\|T\|=\|I-T\|=\frac{1}{2}
$$

then

$$
\frac{\lambda_{n}}{2^{n}} \sum_{k=0}^{n}\left(\begin{array}{l}
n \\
k
\end{array}\right) \frac{1}{\lambda_{k}}=O(1)
$$

implies (2.4).

As any regular triangular generalized method $\mathcal{A}$, satisfying the condition (1.3) can not accelerate the convergence (see [6]), then the following assertion is valid.

Corollary 5. If the conditions (1.7) are satisfied, then the generalized EulerKnopp method $(\mathcal{E}, T)$ can not accelerate the convergence. 
Lemma 5. The conditions

$$
\|T\|=\tau,\|I-T\|=1-\tau, 0<\tau<1
$$

and

$$
\lambda_{n}=O(1)(n+1)^{O(1)}
$$

imply (2.4).

Proof. It follows from (2.7) that conditions (1.7) are satisfied and the condition (2.3) takes a form

$$
\sum_{k=0}^{n}\left(\begin{array}{l}
n \\
k
\end{array}\right) \frac{\lambda_{n}}{\lambda_{k}} \tau^{k}(1-\tau)^{n-k}=O(1) .
$$

The conditions $0<\tau<1$ and (2.8) imply (2.9) (see [15]). It follows from Corollary 3 that (2.4) is valid.

\section{Tauberian Remainder Theorems}

In [13] the first Tauberian theorems for the generalized methods of summability are proved. In [7] Tauberian theorems for semigroups are studied. In [5] statistical Tauberian theorems in metric spaces are examined. In [11] Tauberian conditions, under which statistical convergence follows from statistical summability, are studied. In [15] several Tauberian remainder theorems for Euler-Knopp methods in the case of number matrices and $X=\mathbf{R}$ are proved.

Proposition 2. If the conditions

$$
\begin{gathered}
0<\varphi_{n} \uparrow, \quad \varphi_{n} / n \downarrow \quad\left(n \in \boldsymbol{N}_{0}\right), \\
\lambda_{n} \varphi_{n}\left\|\Delta \xi_{n}^{(\nu)}\right\|=O(1)
\end{gathered}
$$

and (2.3) are satisfied, then

$$
\lambda_{n} \varphi_{n}\left\|\Delta \xi_{n}^{(\nu+1)}\right\|=O(1)
$$

whereas $\xi_{n}^{(\nu+1)}$ is defined by (1.5).

Proof. Using (1.6) we get

$$
\left\|\Delta \xi_{n}^{(\nu+1)}\right\| \leq \sum_{k=0}^{n} \frac{k}{n}\left(\begin{array}{l}
n \\
k
\end{array}\right)\|T\|^{k}\|I-T\|^{n-k}\left\|\Delta \xi_{k}^{(\nu)}\right\| .
$$

Applying (3.4), (2.3) and (3.2) we obtain 


$$
\begin{aligned}
\lambda_{n} \varphi_{n}\left\|\Delta \xi_{n}^{(\nu+1)}\right\| & \leq \sum_{k=0}^{n} \frac{\varphi_{n}}{n} \frac{k}{\varphi_{k}}\left(\begin{array}{l}
n \\
k
\end{array}\right) \frac{\lambda_{n}}{\lambda_{k}}\|T\|^{k}\|I-T\|^{n-k} \\
& =O(1) \sum_{k=0}^{n}\left(\begin{array}{l}
n \\
k
\end{array}\right) \frac{\lambda_{n}}{\lambda_{k}}\|T\|^{k}\|I-T\|^{n-k}=O(1)
\end{aligned}
$$

So the conditions (2.3) and (3.1)-(3.2) imply (3.3).

Corollary 6. The conditions (2.3), (3.1) and

$$
\lambda_{n} \varphi_{n}\left\|\Delta \xi_{n}\right\|=O(1)
$$

imply (3.2), whereas $\xi_{n}^{(\nu)}(\nu \in \mathbf{N})$ is defined by (1.5).

Proposition 3. Let $\nu \in \boldsymbol{N}_{0}$. If $\lambda=\left(\lambda_{k}\right), x^{(\nu)}=\left(\xi_{k}^{(\nu)}\right)$ and $T$ are satisfying the conditions (2.8),

$$
\begin{gathered}
\lambda_{n} \sqrt{n+1}\left\|\Delta \xi_{n}^{(\nu)}\right\|=O(1), \\
(\mathcal{E}, T) x^{(\nu)} \in m_{X}^{\lambda}
\end{gathered}
$$

and $(2.5)$, then $x^{(\nu)} \in m_{X}^{\lambda}$.

Proof. $\quad$ Let $m=[n / 2]$. If

$$
\begin{aligned}
& \rho_{1}(n)=\lambda_{n} \sum_{k=0}^{m}\left(\begin{array}{c}
2 n \\
k
\end{array}\right) T^{k}(I-T)^{2 n-k}\left(\xi_{k}^{(\nu)}-\xi_{n}^{(\nu)}\right), \\
& \rho_{2}(n)=\lambda_{n} \sum_{k=m+1}^{2 n-m-1}\left(\begin{array}{c}
2 n \\
k
\end{array}\right) T^{k}(I-T)^{2 n-k}\left(\xi_{k}^{(\nu)}-\xi_{n}^{(\nu)}\right), \\
& \rho_{3}(n)=\lambda_{n} \sum_{k=2 n-m}^{2 n}\left(\begin{array}{c}
2 n \\
k
\end{array}\right) T^{k}(I-T)^{2 n-k}\left(\xi_{k}^{(\nu)}-\xi_{n}^{(\nu)}\right)
\end{aligned}
$$

and $\rho(n)=\rho_{1}(n)+\rho_{2}(n)+\rho_{3}(n)$ is equal to

$$
\rho(n)=\lambda_{n}\left(\xi_{2 n}^{(\nu+1)}-\xi_{n}^{(\nu)}\right) .
$$

If $0 \leq k \leq m$, then

$$
\xi_{k}^{(\nu)}-\xi_{n}^{(\nu)}=-\sum_{i=k+1}^{n} \Delta \xi_{i}^{(\nu)}
$$

and (3.6) implies

$$
\left\|\xi_{k}^{(\nu)}-\xi_{n}^{(\nu)}\right\|=O(1) .
$$

Therefore using (2.5), (3.9) and Stirling's formula, we get 


$$
\begin{aligned}
\left\|\rho_{1}(n)\right\| & \leq \lambda_{n} \sum_{k=0}^{m}\left(\begin{array}{c}
2 n \\
k
\end{array}\right)\|T\|^{k}\|I-T\|^{2 n-k}\left\|\xi_{k}^{(\nu)}-\xi_{n}^{(\nu)}\right\| \\
& =O\left(n \lambda_{n} 2^{-2 n}\right) \sum_{k=0}^{m}\left(\begin{array}{c}
2 n \\
k
\end{array}\right)=O\left(n^{2} \lambda_{n} 2^{-2 n}\right)\left(\begin{array}{c}
2 n \\
m
\end{array}\right)=O(1) .
\end{aligned}
$$

If $m+1 \leq k \leq 2 n-m-1$, then (3.6) and (2.8) imply

$$
\left\|\xi_{k}^{(\nu)}-\xi_{n}^{(\nu)}\right\|=O(1) \frac{|n-k|}{\lambda_{n} \sqrt{n+1}} .
$$

As

$$
\sum_{k=0}^{m}\left(\begin{array}{c}
2 n \\
k
\end{array}\right)|n-k|=n\left(\begin{array}{c}
2 n \\
k
\end{array}\right)
$$

then using (2.5), (3.10) and Stirling's formula we get

$$
\begin{aligned}
\left\|\rho_{2}(n)\right\| & \leq \lambda_{n} \sum_{k=m+1}^{2 n-m-1}\left(\begin{array}{c}
2 n \\
k
\end{array}\right)\|T\|^{k}\|I-T\|^{2 n-k}\left\|\xi_{k}^{(\nu)}-\xi_{n}^{(\nu)}\right\| \\
& =\lambda_{n} 2^{-2 n} \sum_{k=m+1}^{2 n-m-1}\left(\begin{array}{c}
2 n \\
k
\end{array}\right) O(1) \frac{|n-k|}{\lambda_{n} \sqrt{n+1}} \\
& =O\left(2^{-2 n}\right) \frac{1}{\sqrt{n+1}} \sum_{k=m+1}^{2 n-m-1}\left(\begin{array}{c}
2 n \\
k
\end{array}\right)|n-k|=O(1) .
\end{aligned}
$$

If $2 n-m \leq k \leq 2 n$, then (3.6) implies (3.9). Using (2.8), (2.5), (3.9) and Stirling's formula we get

$$
\left\|\rho_{3}(n)\right\|=O(1) .
$$

Therefore using (3.8) we obtain

$$
\|\rho(n)\|=O(1) .
$$

As

$$
\lambda_{n}\left(\xi_{n}^{(\nu)}-\xi\right)=\lambda_{n}\left(\xi_{n}^{(\nu)}-\xi_{2 n}^{(\nu+1)}\right)+\lambda_{n}\left(\xi_{2 n}^{(\nu+1)}-\xi\right)
$$

while

$$
\lim _{n \rightarrow \infty} \xi_{2 n}^{(\nu+1)}=\xi
$$

then using the conditions (3.11), (3.7) and (2.8) we finish the proof.

Corollary 7. If $\nu \in \mathbf{N}_{0}, \lambda=\left(\lambda_{k}\right), x=\left(\xi_{k}\right)$ and $T$ are satisfying the conditions $(2.8),(2.5)$ and

$$
\lambda_{n} \sqrt{n+1}\left\|\Delta \xi_{n}\right\|=O(1), \quad\left(\mathcal{E}, T^{n}\right) x \in m_{X}^{\lambda},
$$

then $x \in m_{X}^{\lambda}$.

Proof. Using Corollary 6 and Proposition 3 we get step by step the assertion of Corollary 7. 


\section{References}

[1] A. Aasma. Matrix transformations of $\lambda$-boundedness fields of normal matrix methods. Stud. Sci. Math. Hungar., 35, 1999.

[2] S. Baron. Introduction to the theory of summability of series. Valgus, Tallinn, 1977.

[3] J. Boos. Classical and modern methods in summability. Oxford University Press, Oxford, 2000.

[4] C. Brezinski. Numerical analysis 2000. J. Comput. Appl. Math., 122, 1-357, 2000.

[5] J. A. Fridy and M. K. Khan. Statistical theorems in metric spaces. J. Math. Anal. Appl., 282, 744-755, 2003.

[6] I.Tammeraid. Several remarks on acceleration of convergence using generalized linear methods of summability. J. Comput. Appl. Math., 159(2), 365-373, 2003.

[7] B. Jefferies and S. Piskarev. Tauberian theorems for semigroups. Rendiconti del Circolo Math. Di Palermo, 68, 513-521, 2002.

[8] G. Kangro. On matrix transformations of sequences in Banach spaces. In: Proc. Estonian Acad. Sci. Tech. Phys. Math., volume 5, 108-128, 1956. (in Russian)

[9] G. Kangro. Summability factors for the series $\lambda$-bounded by the methods of Riesz and Cesàro. Acta Comment. Univ. Tartuensis, 277, 136-154, 1971. (in Russian)

[10] I. Kornfeld. Nonexistence of an universally accelerating linear summability methods. J. Comput. Appl. Math., 53, 309-321, 1994.

[11] F. Móricz. Tauberian conditions, under which statistical convergence follows from statistical summability $(C, 1)$. J. Math. Anal. Appl., 275, 277-287, 2002.

[12] A. Nappus and T. Sõrmus. Einige verallgemeinerte Matrixverfahren. In: Proc. Estonian Acad. Sci. Phys. Math., volume 45, 201-210, 1996.

[13] T. Sõrmus. Tauberian theorems for generalized summability methods in Banach spaces. In: Proc. Estonian Acad. Sci. Phys. Math., volume 49, 170-182, 2000.

[14] U. Stadtmüller and A. Tali. Comparison of certain summability methods by speed of convergence. Anal. Math., 29, 277-242, 2003.

[15] I. Tammeraid. Tauberian remainder theorems for the Euler-Knopp method of summability. Acta Comment. Univ. Tartuensis, 277, 171-182, 1971. (in Russian)

[16] I. Tammeraid. Convergence rate of iterative process and weighted means. In: Proc. of the OFEA'2001, St. Petersburg, Russia, 2001, volume 2, 49-55, 2002.

[17] I. Tammeraid. Convergence acceleration and linear methods. Math. Model. and Anal., 8(1), 87-92, 2003.

[18] I. Tammeraid. Generalized linear methods and convergence acceleration. Math. Model. and Anal., 8, 329-335, 2003.

[19] I. Tammeraid. Generalized Riesz method and convergence acceleration. Math. Model. and Anal., 9, 341-348, 2004.

[20] K. Zeller. Verallgemeinerte Matrix Transformationen. Math. Z., 56, 18-20, 1952. 